UCRL-ID-120485

\title{
APST Interfaces in LINCS
}

\author{
J.G. Fletcher
}

July 1985

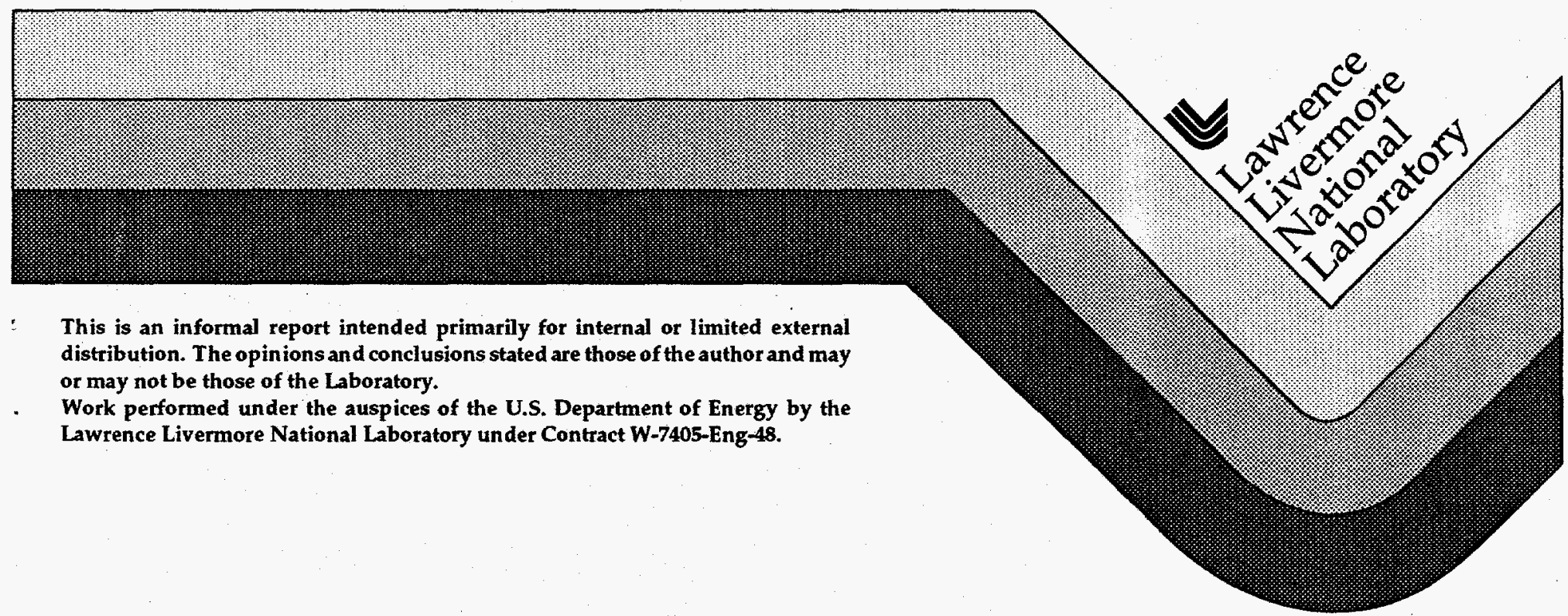

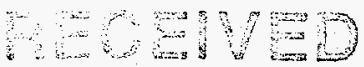

NUL 181025

$09 \mathrm{TI}$ 


\section{DISCLAIMER}

This report was prepared as an account of work sponsored by an agency of the United States Government. Neither the United States Government nor any agency thereof, nor any of their employees, make any warranty, express or implied, or assumes any legal liability or responsibility for the accuracy, completeness, or usefuiness of any information, apparatus, product, or process disclosed, or represents that its use would not infringe privately owned rights. Reference herein to any specific commercial product, process, or service by trade name, trademark, manufacturer, or otherwise does not necessarily constitute or imply its endorsement, recommendation, or favoring by the United States Government or any agency thereof. The views and opinions of authors expressed herein do not necessarily state or reflect those of the United States Government or any agency thereof. 


\section{DISCLAIMER}

Portions of this document may be illegible in electronic image products. Images are produced from the best available original document. 


\title{
APST Interfaces In LINCS
}

\author{
John Fletcher
}

July 23, 1985 
APST Interfaces In LINCS

Table Of Contents

Introduction .3

Functionality of the Layers..... 3

Parameters of the Primitives .. 5

Opening 9

Popen..........................9

Sopen........................... 10

Topen...........................10

Getting Parameters. .11

Pparam 11

Sparam 11

Tparam 11

Sending. 12

Psendc 12

Psendd. 12

Ssend. 12

Tsend. 13

Obtaining 13

Pobtnc 13

Pobtnd 13

Sobtn: 14

Tobtn 14

Giving 15

Pgivec. 15

Pgived 15

Sgive. 15

Tgive 15
Receiving 16

Precve 16

Precvd.......................... 16

Srecv 17

Trecv .17

Getting Errors. 17

Perror. 17

Serror. 18

Terror 18

Canceling.. 18

Pcancl.......................... 18

Scancl.......................... 19

Tcancl 19

Aborting... 19

Pabort 19

Sabort. 19

Tabort. 19

Closing 20

Pclose.........................20

Sclose..........................20

Tclose...........................20

Initializing.............................20

Pinit............................20

Sinit...........................20

Tinit.............................20

Errors ................................... 21

Interaction with SMILE ...........22

Index..................................... 23 
APST is an acronym for the four highest of the seven layers of the LINCS hierarchy of communication protocols: (from high to low) Application, Presentation, Session, and Transport. Routines in each but the lowest of these APST layers can utilize the facilities of any lower APST layer (normally, but not necessarily, the immediately next lower layer) by invoking various primitives (macros that in most cases are subroutine calls) defining the upper interface of the lower layer. So there are three APST interfaces: Presentation layer, used by the Application layer; Session layer, normally used by the Presentation layer; and Transport layer, normally used by the Session layer. The present document defines these three interfaces.

Logically, each end of a stream (unidirectional sequence of transmitted information) is handled by three modules, one module each for the Presentation, Session, and Transport layers, and each of these modules deals with only that one end of that one stream. The internal workings of the layers, particularly the Transport layer, do not necessarily exhibit this same modularization; for example, the two oppositely directed streams between the same two ends (constituting an association) may interact within a layer. However, such interaction is an implementational detall of no direct interest to those utilizing the layer. The present document does not describe implementation, nor does it discuss in any detail how the modules employ packet headings and data formats to communicate with their partner modules at the other end of a stream.

There being one logical module per end of stream is a characteristic only of the Presentation, Session, and Transport layers. An Application layer module usually manages several streams, orchestrating them to achieve some desired purpose. The modules of the layers (Network, Link, and Physical) below the APST layers each handle many streams, multiplexing them through the nodes and channels of the network to transmit them from their origins to their destinations; also, the interfaces to those layers are of a somewhat different character from the APST interfaces discussed here.

The APST primitives, here defined as they appear in the $\mathrm{C}$ language, are built upon the SMILE primitives, defined elsewhere, with which the reader is assumed to be familiar.

\section{Functionality of the Layers}

A Transport layer module sends or receives information to or from a partner Transport layer module at the other end of a stream. The transmitted information consists of two kinds of data bits -- 0 and 1 -and, interspersed among the data bits (usually sparsely). three kinds of marker bits -- B (begin), W (wake), and $\mathbf{E}$ (end) -- each bit being labeled with a protection level. Marker bits are passed across the Transport layer's (upper) interface as parameters or values of certain primitives, while data bits are passed in buffers, a buffer being a block of zero or more consecutive data bits, all of the same protection level. Buffers are (8-bit) byte oriented. Each consecutive block of eight bits constitutes a byte, with the high-order (most significant or leftmost) end of the byte (no matter how the bits within the byte are numbered on a particular computer) preceding 
the low-order (least significant or rightmost) end. (This treatment of bits within a byte applies to all bytes handled by the APST Primitives, not just to bytes in buffers.) If the number of bits in a buffer is not an integral multiple of eight, the low-order end of the last byte is unused. The bytes of a buffer are ordered according to the addressing of the computer; so, unlike bits within a byte, bytes within a computer word proceed either left to right or right to left. depending upon whether the computer is a "big end" or a "little end" computer.

The Transport layer guarantees (with a suitably high probability) delivery assurance, that is, that all bits sent are received, without omission, duplication, missequencing, or corruption. It attaches no interpretation to the bits; so the Transport layer is used for the reliable transmission of "raw" Information. Transport layer primitives all are named beginning with an upper case " $T$ ".

The Session layer enhances the Transport layer by imposing the LINCS monolog protocol on the marker bits. This protocol treats a stream as a sequence of monologs, each monolog delimited by a B-bit at its beginning and an E-bit at its end. The Session layer enforces the rule that no bits should occur between an E-bit and the following B-bit and that B-bits and E-bits should alternate: it also enforces the rule that an entire monolog should be of a single protection level. The W-bits are separators that divide a monolog in to messages, each message being bounded at each end by a (B, W, or E) marker bit. The primary purpose of sending W-bits is to wake up the receiver: the receiving Transport layer will return control to the Session (or higher) layer only when a buffer fills and/or a W- or E-bit is received. Session layer primitives all are named beginning with an upper-case "S".

The Presentation layer enhances the Session layer by providing an interpretation to the data bits. Two Presentation layer protocols are available: data and control. The data Presentation protocol treats the data bits as just a string of bits; that is, it does not interpret them. The chief difference between the Presentation and Session layers in the case of the data protocol is that a receiving Presentation layer module discards $\mathbf{W}$ bits, because W-bits are intended only to effect wake up, not to convey meaning.

The control Presentation protocol provides an interface that permits the Application to send and recelve functions and their parameters. Logically, the Application should "think" in much the same manner as when it invokes a subroutine: it is providing parameters to another module, or it is getting parameters back. It does not need to know that the parameters are encoded by the sending Presentation layer into strings of bits called tokens and then decoded by the receiving Presentation layer. Presentation layer primitives all are named beginning with an upper-case "P".

The Application layer utilizes the primitives of the Presentation layer (and, if it wishes, the Session and/or Transport layers) to achieve whatever purposes it may have. There are no restrictions on its behavior, out it must understand the functionality of each primitive that it uses if it expects to obtain coherent behavior. 
All primitives are integer-valued. The value indicates the success (zero and positive values) or failure (negative values) of the primitive and the nature of that success or failure. For all except the primitives effecting the receiving of information, the only success value is zero. The receiving Primitives indicate success by making use of the following end-code values, which are also used for other purposes; each value is defined by a symbolic constant, the actual value of which is also shown:

- Ebit (1) E-bit (end monolog);

- Wbit (2) W-bit (message boundary or wakeup);

- Nbit (4) Transmit now (not used in the value of any primitive);

- Bbit (8) B-bit (begin monolog).

(As is the case for all values defined by symbolic constants, the actual values are not guaranteed; so it is strongly recommended that only the symbolic forms be used.) These values have been chosen so that they can be or-ed together to indicate several things at once. So a receiving primitive can return data bits and/or one or more marker bits; a returned value of zero indicates no marker bits. Failure values range from -1 to 255 and are the negatives of standard LINCS errors. They can indicate parsing errors, resource overloads, etc., and are listed below.

The parameters passed to the various primitives are drawn from a list of about a dozen and half kinds of parameters, now discussed. Each Parameter is here given a mnemonic (which has no official standing), and its C-language type (C-type) is indicated.

$\begin{array}{lll}\text { char * } & \text { locaddr } & \text { (local network address), } \\ \text { char } & \text { remaddr } & \text { (remote network address), and } \\ \text { char * } & \text { streamno } & \text { (stream number) }\end{array}$

are used to define a stream. Each is a pointer to an array of eight bytes that contains the address or stream number; the most significant byte of the address or stream number is byte 0 of the array. (The number of bytes in an address array may differ from eight in certain special situations involving non-LINCS protocols.) The notion of "any" in defining one of these parameters for a stream is indicated by using a Null pointer.

$$
\text { char level ' (protection level) }
$$

is an integer indicating a protection level. Only values less than 8 are in use.
char
mode
(mode)

is used to define certain characteristics of a stream. The following bitencoding is used, and the values may be or-ed together as appropriate:

- Mlob (1) Buffers for a data stream are to be provided by the

- Mcon (2) The stream is control, not data;

- Mrcr (4) The stream is receiving, not sending;

- Mmul (8) The stream is for multiple monologs, not just one.

char * buffer (buffer pointer) 
is a pointer to an array constituting a buffer for data bytes. The bytes are stored in the order transmitted, earlier bytes having lower array indices.

\begin{tabular}{|c|c|c|}
\hline $\begin{array}{l}\text { int } \\
\text { int } \\
\text { int } \\
\text { long } \\
\text { long }\end{array}$ & $\begin{array}{l}\text { count } \\
\text { size } \\
\text { window } \\
\text { unacked } \\
\text { unsent }\end{array}$ & $\begin{array}{l}\text { (buffer count), } \\
\text { (buffer size). } \\
\text { (window size), } \\
\text { (unacked count). } \\
\text { (unsent count). }\end{array}$ \\
\hline
\end{tabular}

are used in various contexts (discussed below) to indicate the number of bits in a buffer, in a window, or involved in an error situation. Negative values are permitted, because in some cases (discussed below) the negative memory-block-size code avallable in SMILE can be used. It is assumed that the user finds it more convenient to restrict sizes and counts to those than can be expressed as an integer; so the long type is used only for parameters associated with error conditions (where the value may exceed the size of the largest buffer. It is important to note that in all cases bits, not bytes, are being enumerated; this is in contrast with SMILE primitives, where bytes are enumerated.

$$
\text { char end (end code) }
$$

is used to define the marker bits involved in a transmission. The same end-code values (above) are used as in the returned values of receiving primitives; they may be or-ed together.

$$
\text { char * mor (monolog control record pointer) }
$$

is a pointer to an array that holds the parameters involved in a control transmission. (These parameters are not to be confused with the parameters of the primitive that effects the transmission.)

$$
\text { struct tmap * map (token map pointer) }
$$

is a pointer to an array constituting a map that describes what parameters are being sent or received in a control transmission. Each element in the array is a structure that defines one parameter as follows:

$\begin{array}{cl}\text { struct tmap ( } & \\ \text { char } & \text { mflg; } \\ \text { char } & \text { mtyp; } \\ \text { char } & \text { musg; } \\ \text { char } & \text { ming; } \\ \text { int } & \text { mval; \}; }\end{array}$

The members of this structure are used as follows:

- char mflg (flag) is a bit vector encoded as follows:

- Ftrn (l)

Indicates that a recelved parameter may be truncated (and the truncated bytes discarded) without error.

- Fimm (2) Indicates that the value of a parameter being sent, not a pointer to the value, is in the map. This flag is intended to be used for sending constant parameters (that are short enough to fit into the map).

- Find (4) Indicates that the address of a parameter being sent is in the map. 

parameter is absolute, not relative to the pointer to the monolog control record. The absolute option is intended for global parameters shared by many tasks. The relative option provides a way for several tasks to share a fixed, reentrant map yet each have its own local parameters: the monolog control record pointer then differs from task to task and points to a structure peculiar to each task (such as to a place on its "stack").

- Fetc $(0 \times 40)$ Indicates to ignore any extra tokens.

- Fend $(0 \times 80)$ Indicates the last entry in the map. (It does not follow the last entry.)

- char mtyp

(type) indicates the type of the parameter. The defined types are as follows:

- Tnat (0)

- Tint (1)

- Tbit (2)

- Tcap (3)

- Tchr (4)

- Traw (5)

- Tvar (0x80) Variable type.

When receiving, Trar (the high-order bit of the type) may be set to indicate a variable type; the rest of the type field is then ignored, and the type is stored with the value (as described below).

- char musg

- char ming

- int mval is, the purpose of the parameter in the operation (usage) indicates the usage of the parameter, that being performed (in contrast with subroutine calls in most languages, where purpose is usually indicated by position). If the usage is 0 , then the entry is ignored, except that whether or not it is the last entry is noted; it is often convenient to make the last entry one with a 0 usage, and so doing is the only way to form a map with effectively no entries.

(length) is the number of bytes (not bits) in the array of bytes containing the value. Zero is permitted as a length (and implies a zero value or empty string).

(value) defines the value of the parameter. No matter what C-type (not to be confused with the control protocol type defined above) a parameter is, the primitives treat it as an array of characters (their number being equal to the length). So the appropriate $\mathrm{C}$-type of mval depends on whether the value is immediate (Fimm or-ed into the flag), 
absolute (Fabs or-ed into the flag). or relative (neither Fimm nor Fabs or-ed into the flag); int is appropriate only in the last case. when mval is added to the monolog control record pointer. (The monolog control record pointer may be Null if no relative values occur in the map.) Casting is normally used to cope with the other two cases. where the appropriate type are char * (absolute) and char [sizeof(int)] (immediate).

Values for type Tchr (4) and Traw (5) are padded or truncated at the least significant end and for all other types at the most significant end. For types Tcap (3). Tchr (4), and Traw (5) the most significant end is placed into the lowest computer address and for all other types as appropriate to the computer (the same as for types Tcap (3), Tchr (4) and Traw (5) on "big end" computers, oppositely on "little end" computers). If the type is variable when receiving and is therefore to be stored with the value, it is always stored at the lowest computer address (where the pointer points), and then the next higher byte address is used as defining the location of the value: the length does not include the byte for the type.

unsigned int tokcount (token count)

is the number of parameters received by an invocation of the receiving primitive on a control stream.

int error (error)

is an error code returned by the getting-error primitives to define what kind of transmission error occurred. The value returned by a getting-error primitive indicates the success or failure of that primitive to do its action and is not to be confused with the returned error parameter.

When * flag (reject flag)

is used by all primitives (except the initializing primitives) to indicate whether or not the operation of the primitive is to be rejected (terminated early before completion, as with a tape player; the word "abort" is not used to describe this action, because that word is used below for another purpose). The various primitives may internally go to Sleep (by calling the SMILE Sleep primitive) waiting for some external event (such as the receipt of transmitted information) that is necessary to the completion of their purpose. There must be some way to guard against indefinite waiting (such as for information that will never be received). The reject flag provides for two kinds of premature termination: timeout and intervention by another task. The reject flag is a pointer to a time interval. Each primitive will internally wait no longer than this time interval. Intervention by another task to effect rejection is accomplished by setting the interval to zero and then Waking the task that is to experience the rejection. A Null reject flag is treated as if pointing to zero and inhibits waiting.

Sid id (stream identifier)

identifies the stream on which a primitive is to operate. It is returned when a Presentation layer stream is opened and then is used with 
subsequent invocations of primitives relating to that stream. The C-type Sid is implementation-dependent and defined as part of the APST package. A Null (zero) stream identifier indicates no stream and is acceptable to some of the primitives (as discussed below).

Parameters can be used either to supply information to a primitive or to get information back. In the latter case, the parameter is a pointer to where the information is to be placed (so an extra *usually occurs in its C-type), and here "_ret" is appended to its mnemonic. Some parameters (e.g., token count, error) occur only in this form. A Null pointer indicates that the particular piece of returned information is not wanted. Even if a primitive fails, the returned items all have appropriate values, often Null or zero.

Each Primitive is named with an initial upper-case letter (P, S, or T) defining its layer, followed by four or five lower-case letters defining its function. Similar functions in each of the three layers use the same lower-case letters in their names and are here discussed together.

\section{Opening}

Popen ( locaddr, remaddr, streamno, level, mode, window, id_ret, flag )

$\begin{array}{ll}\text { char * } & \text { locaddr; } \\ \text { char * } & \text { remaddr; } \\ \text { char * } & \text { streamno; } \\ \text { char } & \text { level; } \\ \text { char } & \text { mode; } \\ \text { int } & \text { window; } \\ \text { Sid * } & \text { id_ret; } \\ \text { When * } & \text { flag; }\end{array}$

opens a stream for action by other primitives and returns an identifier id ret for the use of those primitives. The stream is defined by local address *locaddr, remote address *remaddr, and stream number "streamno; any or all of these pointers may be Null, indicating "any". Whether the stream is data or control and whether it is to be sending or receiving is indicated by the bits in mode. The other bits in mode and the window (used only for a receiving data stream) are used as described below for Topen. The level is the protection level of the bits in the stream when sending and a maximum for that level when receiving. If id_ret is Null, a no-op is performed.

Sopen ( locaddr, remaddr, streamno, level, mode, window, id, flag )

$\begin{array}{ll}\text { char * } & \text { locaddr; } \\ \text { char * } & \text { remaddr; } \\ \text { char * } & \text { streamno; } \\ \text { char } & \text { level; } \\ \text { char } & \text { mode; } \\ \text { int } & \text { window; } \\ \text { Sid } & \text { id; } \\ \text { When * flag; }\end{array}$

opens a stream for action by other Session and Transport layer primitives. The parameters are used as with Popen, except that the mode bit 
Mcon indicating control vs. data is ignored, and the stream identifier is supplied to the Primitive, not returned by the primitive. That is, the identifier is selected by the Presentation layer (or the Application layer. provided that it knows how) and then used by the lower layers. This places some constraints on reasonable implementations of stream identifiers, but the following are, for example, quite feasible: the identifier is an array index locating (probably in separate arrays) record structures used by the layers, or the identfier is a pointer to a structure such that lower layer structures are embedded in higher layer structures.

Topen ( locaddr, remaddr, streamno, mode, window, id, flag )

$\begin{array}{ll}\text { char * } & \text { locaddr; } \\ \text { char } & \text { remaddr; } \\ \text { char } & \text { streamno; } \\ \text { char } & \text { mode; } \\ \text { int } & \text { window; } \\ \text { Sid } & \text { id: } \\ \text { When * flag; }\end{array}$

opens a stream for action by other Transport layer primitives. The parameters are used as with Sopen, except as now noted. The Transport layer prepares to allocate data buffers as needed, if the mode bit Mlob indicates that it is to do so or if the mode bit Mcon indicates a control stream (since in the latter case it is assumed that the Presentation layer -- which gets monolog control records and token maps, not data buffers, from the Application layer -- does not provide the data buffers). If it is to allocate buffers for a receiving stream the Transport layer immediately does so by providing capacity at least equal to the window, which can be expressed using a negative memory-size code times 8 (to convert bytes to bits). as well as with a nonnegative bit count. The multiple monolog bit Mmul in the mode is used to determine whether or not transmission on the stream is to be automatically shut down upon encountering an $\mathbf{E}$ bit. There is no level parameter, because the Transport layer treats protection levels on a buffer-by-buffer basis.

\section{Getting Parameters}

Pparam ( id. locaddr_ret, remaddr_ret, streamno_ret, level_ret, flag Sid ld:
char * locaddr_ret;
char * remaddr_ret;
char $*$ streamno_ret:
char * level_ret;
When * flag: :

returns the local address, remote address, stream number, and protection level associated with a stream. (If any of these are not wanted, Null pointers may be used.) Note that the two addresses and the stream number are placed into arrays pointed to by the passed parameters (rather than, as might have been supposed, having a pointer to an array 
placed where the parameter points). Note also that here is a third use of the term "parameter", describing characteristics of a stream, rather than information passed to a primitive or information transmitted in a control stream. Pparam will wait until the addresses, stream number, and level are known (or until a reject) before returning. The addresses and/or stream number are not known if they were originally "any" and are not yet specific, and the level is unknown between receiving monologs. A special case is that a Null (zero) stream identifier can be used, in which case the returned items are as follows: the local address is one that the invoking task can use; the remote address is that of the local location server; the stream number is a random number; and the protection level is the highest usable by the invoking task.

Sparam ( id, locaddr_ret, remaddr_ret, streamno_ret, level_ret, flag

Sid id;

char * locaddr_ret;

char * remaddr_ret;

char * streamno_ret;

char * level_ret;

When * flag;

is identical to Pparam.

Tparam ( id, locaddr_ret, remaddr_ret, streamno_ret, flag )

Sid id;

char * locaddr_ret;

char * remaddr_ret;

char* Streamno_ret;

When * flag;

is identical to Pparam and Sparam, except that it does not return a protection level (since the Transport layer does not regard a stream as having a level).

\section{Sending}

Psendc ( id, mcr, map, end, flag )

$\begin{array}{ll}\text { Sid } & \text { id; } \\ \text { char * } & \text { mcr; } \\ \text { struct tmap * } & \text { map; } \\ \text { char } & \text { end; } \\ \text { When * } & \text { flag; }\end{array}$

sends information on a control stream. The parameters defined by the map are sent in the order that they appear in the map and are terminated by a single "go" indicator to indicate the end of a statement. A map with no entries (except usage 0 entries that are ignored) effects the sending of no parameters, just a "go". However, a Null map pointer suppresses all transmission (not even a "go" is sent), other than marker bits. The end parameter indicates what marker bit (W or $\mathbf{E}$ only), if any, 
is to be sent following the "go". if any. The parameters and "go" are not necessarlly sent immediately; however. they are sent immediately if followed by a $\mathbf{W}$ or $\mathbf{E}$ or if "transmit now" is indicated in the end code. It is intended that in most cases the map used by a Psendc be a constant structure, generated once, preferably when the program is compiled or if necessary during an initialization phase; then, just as in the case of subroutine invocation, all that changes between successive invocations of a Psendc are the parameter values.

Psendd (id. buffer, count, end. flag )

$\begin{array}{ll}\text { Sid id: } & \text { idf } \\ \text { char } & \text { buffer; } \\ \text { int } & \text { count; } \\ \text { char end; } & \text { When } \\ \text { flag; }\end{array}$

sends information on a data stream. The information is defined by buffer and count and may be followed by $\mathbf{W}$ or $\mathbf{E}$ as indicated by end; sending is necessarily immediate only if $\mathbf{W}, \mathbf{E}$, or "transmit now" is indicated. A zero count buffer and/or a Null buffer pointer suppress data (but not marker bit) transmission. Completion of any of the send primitives does not mean that the information has been sent but only that the lower layer has accepted responsibility for the sending; this is so even when immediate sending is being attempted.

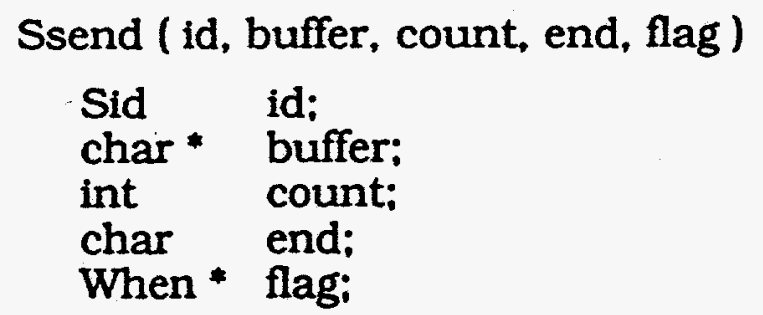

is Identical to Psendd.

Tsend (id, buffer, count, end, level, flag)

$\begin{array}{ll}\text { Sid } & \text { id: } \\ \text { char } & \text { buffer; } \\ \text { int } & \text { count; } \\ \text { char } & \text { end; } \\ \text { char level; } & \text { When * flag; }\end{array}$

sends information on a stream. The differences from Psendd and Ssend are that the end code can indicate a B-bit (which precedes, rather than follows, any data), as well as a W. E. or "transmit now", and that a protection level for all bits sent must be indicated. Also Psendc, Psendd, and Ssend, unlike Tsend, are designed to combine information from successive invocations before transmitting it. In particular, a data buffer without following marker bits presented in one invocation will be held and combined with marker bits presented with a Null data buffer in the following invocation. That is, the sending Presentation and Session layer primitives are oriented so as to work efficiently on behalf of Applications 
that do not pass data and marker bits in the same invocations, a

behavior which in many cases is the most natural way to program. On the other hand, Tsend is not intended to be so oriented and will typically transmit as a unit whatever is delivered to it in a single invocation.

\section{Obtaining}

Pobtnc (id, mcr_ret, flag )

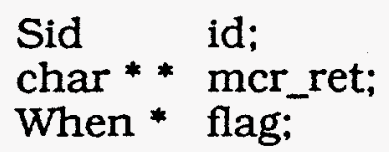

waits until the parameters defined in the preceding Psendc have been entirely fetched from memory and then returns; only after Pobtnc has been executed can the parameters safely be modified. Every Psendc must be followed by a Pobtnc before another Psendc can be executed, except when the former Psendc used a Null map pointer. Pobtnc returns a pointer to the monolog control record. Unnecessary executions of Pobtnc are no-ops returning Null monolog control record pointers.

Pobtnd (id, size, buffer_ret, count_ret, flag )

$\begin{array}{ll}\text { Sid } & \text { id; } \\ \text { int } & \text { size; } \\ \text { char * * } & \text { buffer ret; } \\ \text { int * } & \text { count ret; } \\ \text { When * } & \text { flag; }\end{array}$

returns a pointer to a data buffer used for sending and a count for that buffer. There are two cases: If the Application layer supplies the buffers, then Pobtnd reacquires a buffer used in a preceding Psendd. Unlike the case of a control stream, a Pobtnd does not have to occur between every pair of Psendd invocations; that is, several Psendd invocations can be made before the first Pobtnd, up to a limit that is implementation dependent. This limit can be "experimentally" determined by sending until a sequence error (discussed below) occurs; if the limit is one, then the efficiencies of double buffering should be provided internally by the Presentation (or lower) layer. Buffers are returned in the same order as sent, and the returned count is the same as for the send. The only exception to all this is that a Null (but not a non-Null zero-count) buffer is never returned and no attempt to obtain it should be made. The second case is that the Transport layer supplies the buffers. In this case Pobtnd precedes the corresponding Psendd. The buffer returned is at least as large as is specified by size (which can be a negative memory size code times 8 ); the size is ignored when the Application layer supplies the buffers. A Null buffer is never obtained (even when the requested size is zero), and buffers should be sent in the same order as obtained; after all obtained buffers have been sent, Null buffers may be sent. The implementation may allow getting ahead by some number of obtains. Successfully obtaining a buffer, even when originally supplied by the Application layer, does not necessarily mean that the data in the buffer has been sent, since it may have been internally copied by a lower layer. 
Sobtn (id, size, buffer_ret, count_ret, flag )

Sid id:
int $\quad$ size:
char - buffer_ret:
int * count_ret:
When * flag;

is identical to Pobtnd.

Tobtn (id, size, buffer_ret, count_ret, flag )

$\begin{array}{ll}\text { Sid } & \text { id; } \\ \text { int } & \text { size; } \\ \text { char - } & \text { buffer_ret; } \\ \text { int * count_rct; } & \text { con } \\ \text { When * flag; }\end{array}$

is essentially identical to Pobtnd and Sobtn; there are only such subtle differences as derive from the fact that Psendd and Ssend, unlike Tsend, when possible combine data and marker bits from successive invocations before transmitting them.

\section{Giving}

Pgivec (id, mcr, map, flag )

$\begin{array}{ll}\text { Sid } & \text { Id; } \\ \text { char } & \text { mcr; } \\ \text { struct tmap } & \text { map; } \\ \text { When } & \text { flag; }\end{array}$

provides a monolog control record and a token map to be used to receive parameters on a control stream. As with Psendc, it is intended that in most cases the map used by Pgivec be a constant structure. Giving and receiving are related in the same way as are sending and obtaining: giving and sending supply monolog control records and tokens maps (or. for data streams, buffers) to the lower layers, while receiving and obtaining get them from the lower layers. So waiting normally occurs when receiving or obtaining. Overlap of transmission and computation may be achieved by computing between a give or send and the following receive or obtain.

Pgived (id, buffer, count, flag )

Sid id:
char * buffer;
int count;
When * flag;

provides a buffer of count bits to be used for a subsequent receive (if the Application layer supplies the buffers) or returns to the lower layers a buffer that contained previously received information (if the Transport layer supplies the buffers). A zero count buffer is usable only for 
receiving marker bits. A Null buffer pointer is useless, causing an effective no-op, and no receive corresponding to it should be invoked.

Sgive (id, buffer, count, flag )

$\begin{array}{ll}\text { Sid } & \text { id; } \\ \text { char * buffer; } \\ \text { int } & \text { count; } \\ \text { When * flag; }\end{array}$

is identical to Pgived.

Tgive ( id, buffer, count, flag )

$\begin{array}{ll}\text { Sid } & \text { id; } \\ \text { char * } & \text { buffer; } \\ \text { int } & \text { count; } \\ \text { When * flag; } & \end{array}$

is identical to Pgived and Sgive.

\section{Receiving}

Precve (id, mcr_ret, tokcount_ret, flag)

$\begin{array}{ll}\text { Sid } & \text { id; } \\ \text { char * } & \text { mcr_ret; } \\ \text { unsigned int * } & \text { tokcount_ret; } \\ \text { When * } & \text { flag; }\end{array}$

receives the parameters in one statement (up to a "go") on a control stream. The token map and monolog control record used are those supplied by a preceding Pgivec. Each parameter received is stored as indicated by the map entry of the same usage. A map with no entries (except usage 0 entries that are ignored) can be used to receive a statement with no parameters (just a "go"). However, a Null map, which is assumed after each Precve until a Pgivec is invoked, is effective only for receiving marker bits (not even for a "go"). Parameters and marker bits are returned separately, and each marker bit (E only) is returned by itself; that is, the end of monolog marker is received, not by the same invocation of Precve that receives the last statement of the monolog, but by the next following invocation of Precve. The monolog control record pointer of the preceding Pgivec is returned with the parameters; Null is returned in its stead with a marker bit. The returned token count is the number of parameters processed plus 1 (for the "go"); 0 is returned in its stead with a marker bit.

Precvd (id, buffer_ret, count_ret, flag )

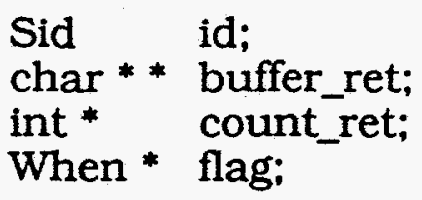


receives one buffer of data bits, the buffer pointer and number of bits being returned. There are two cases: If the Application layer supplies the buffers, then the buffer returned was provided with a preceding Pgived. Several Pgived invocations, up to a limit dependent on the implementation, may be given before the first Precvd. This limit may be learned "experimentally" as with Psendd and Pobtnd. and the efficiencies of double buffering should be effectively available, even when the limit is one. Buffers are received in the same order as given. The second case is that the Transport layer supplies the buffers. In this case Precvd precedes the corresponding Pgived. Buffers should be given back in the same order as received, except that received Null (but not non-Null zerocount) buffers need not be given back (in fact, given Null buffers are ignored). The implementation may allow getting ahead by some number of receives. In either of the two cases, data buffers are received only when the returned value of the primitive is zero. As with Precvc, marker bits (E only) are returned individually, in association with a Null buffer pointer and a zero count.

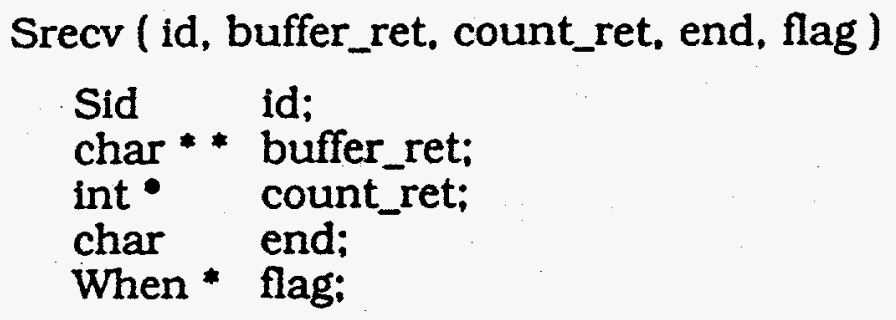

differs from Precvd only in the following: The returned value may indicate $\mathbf{B}$ or $\mathbf{W}$, as well as $\mathbf{E}$ or data. The parameter end is bit-encoded to indicate $\mathbf{B}, \mathbf{W}$, and/or $\mathbf{E}$; the indicated bits are inhibited; that is, if encountered, they are processed and discarded by the Session layer out not returned in the value. The "transmit now" bit is used to indicate that any data bits are to be discarded (the returned buffer count is zero).

Trecv ( id. buffer_ret, count_ret, level_ret, flag )

Sid id;
char.* buffer_ret;
int ${ }^{*}$ count_ret;
char * level_ret;
When * flag;

differs from Precvd and Srecv as follows: Marker bits and data are not necessarlly returned separately. A non-Null data buffer can be returned even when the returned value is positive, and $B, W$, and $E$ can be or-ed together in the returned value: the $B$ is understood to precede, and $W$ or $\mathbf{E}$ to follow, the data (if any). There is no inhibition of returned marker bits. A protection level, which applies to all returned bits, is also returned. If the Application layer supplies the buffers, nothing can be received unless a buffer (possibly an empty buffer if no data is expected) has been given; if the Transport layer supplies the buffers, a Null buffer is never received. 
Perror ( id, error_ret, unacked_ret, unsent_ret, flag )

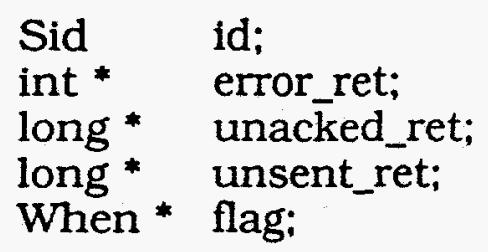

is used to determine the nature of any transmission error that may have occurred on a stream. Transmission errors are ones that occur on a stream during the process of transmission by the Transport layer, asynchronously with execution of the primitives. Such an error constitutes a failure in the delivery assurance guarantee of the Transport layer; namely, transmitted information may have been lost. Transmission errors typically are detected by the inner mechanisms of the Transport layer timing-out when waiting for a response; such timing out is best known as a sending phenomenon cut is also possible when receiving. When the error occurs, the stream goes into an error state such that any primitive, except one of the getting-error primitives, returns the (unique) transmission error value (discussed below). The error state can be cleared only by executing Perror, Serror, or Terror;. These primitives return an implementation-dependent error code indicating the nature of the transmission error; this code is not to be confused with the returned value of the error primitive, which indicates whether or not the primitive succeeded in its operation (which it usually does). Also returned are the number of bits sent but not acknowledged and the number of bits accepted for sending but not sent; these returns are both zero for a receiving stream. If a getting error function is invoked when the stream is not in an error state, the primitive will wait until an error state is entered or until it is certain that zero can be returned for both of the counts; in the latter case the returned error code is zero. Applied to an invalid stream identifier, Perror is a successful no-op.

Serror (id, error_ret, unacked_ret, unsent_ret, flag )

$\begin{array}{ll}\text { Sid } & \text { id; } \\ \text { int * } & \text { error_ret; } \\ \text { long * } & \text { unacked_ret; } \\ \text { long * } & \text { unsent_ret; } \\ \text { When * } & \text { flag; }\end{array}$

is identical to Perror.

Terror ( id, error_ret, unacked_ret, unsent_ret, flag )

$\begin{array}{ll}\text { Sid } & \text { id; } \\ \text { int } & \text { error_ret; } \\ \text { long * } & \text { unacked_ret; } \\ \text { long * } & \text { unsent_ret; } \\ \text { When * } & \text { flag; }\end{array}$

is identical to Perror and Serror. 


\section{Canceling}

Pcancl (Id, flag)

Sid id:

When * flag:

when sending causes the lower layers not to attempt to send any bits that they have accepted for sending but have not as yet attempted to send. Marker bits are forgotten, and buffers of data bits treated as though shortened to include only those bits (possibly none. possibly all) already sent; these buffers must still be obtained back if supplied by the Application layer. When receiving. Pcancl causes the lower layers not to use any buffers or part of a buffer that they have been given but have not as yet filled with bits. These buffers are appropriately shortened and must be received back if supplied by the Application layer. For control streams sent or given token maps and monolog control records are replaced by Nulls. Canceling is intended for use when an Application "changes its mind". Since canceling strikes somewhat unpredictably in the midst of transmission, its effect, particularly on control streams, is not always knowable; severe damage can occur to a stream being sent. damage from which recovery can be quite difficult. Applied to an invalid stream identifier, Pcancl is a successful no-op.

Scancl (id, flag )

Sid id;

When * flag;

differs from Pcancl only in that it has no effect on token maps and monolog control records.

Tcancl (id. flag )

Sid id:

When * flag;

is identical to Scancl.

\section{Aborting}

Pabort (1d, flag )

Sid id:

When * flag;

: when sending, unless the last bit submitted for sending was an E-bit, sends an E-bit; that is, it quickly terminates the monolog (if any). When receiving. Pabort discards all bits received up to and including the next E-bit (if the last bit received before the abort was an E-bit, then nothing is discarded); that is, it makes certain that a monolog has ended. Also, logically subsequent to the end monolog. a cancel is effected. Applied to an invalid stream identifier, Pabort is a successful no-op. 
Sabort (id, flag )

Sid id;

When * flag;

is identical to Pabort.

Tabort (id, end, flag )

$\begin{array}{ll}\text { Sid } & \text { id; } \\ \text { char end; } & \text { entag; }\end{array}$

differs from Pabort and Tabort in that the parameter end specifies which bit (W as well as $\mathbf{E}$ and, for receiving, B) terminates the action. A received terminating $\mathbf{W}$ or $\mathbf{E}$ is discarded, but a received terminating $\mathbf{E}$ ) is not.

\section{Closing}

Pclose (id, flag)

Sid id;

terminates activity on a stream by invoking Sclose and then discarding any records associated with the stream identifier. The identifier is then available for reuse when a subsequent Popen is invoked.

Sclose (id, flag)

Sid id;

When * flag;

terminates activity on a stream by first aborting the stream and then invoking Tclose.

Tclose (id, flag )

Sid id;

When * flag;

terminates activity on a stream by bringing transmission to a halt (for . sending, this means sending all bits that have been accepted for sending and making sure that they are acknowledged). It is then assumed that all buffers are returned to the layer (Application or Transport) that supplied them; no further sending, obtaining, giving, or receiving is needed or in fact is possible. The only thing that can make it impossible to close is that the stream is in a transmission error state. (A loop consisting of closing and getting error will soon effect a close.) Applied to an invalid stream identifier, any of the closing primitives are successful no-ops. 
Pinit ()

initializes the Presentation layer and invokes Sinit. It should be invoked exactly once before any other Presentation layer primitive is invoked.

Sinit ()

initializes the Session layer and invokes Tinit. It should be invoked exactly once (usually by Pinit) before any other Session layer primitive is invoked.

Tinit ()

initializes the Transport layer. It should be invoked exactly once (usually by Sinit) before any other Transport layer primitive is invoked.

\section{Errors}

The above primitives can exhibit the following errors, identified by symbolic constants beginning with an upper-case " $\mathrm{X}$ ".

Xbgn (-8) begin error, is returned when Precvc, Precvd, or Srecv encounters a B-bit. other than immediately following an E-bit or immediately after the stream is opened.

Xlsn (-9) listen error, is returned when Precvc, Precvd, or Srecv does not encounter a B-bit as the first bit of a stream or as the bit immediately following an E-bit.

Xend (-12) end error, is returned by Precve when it encounters a W or E-bit, other than immediately following a "go".

Xlch (-14) level change error, is returned when Precvc, Precvd, or Srecv encounters a change in the protection level of the bits in a stream, other than coincident with a Bbit.

Xsep (-34) separator error, is returned by Precve when a "go" is improperly encoded.

Xusg (-35) usage error, is returned by Precve when it encounters a parameter with a usage not provided for in the token map.

Xtyp (-36) type error, is returned by Precve when it encounters a parameter with a type other than indicated in the token map.

Xval (-37) value error, is returned by Precve when it encounters a value for a parameter which cannot be fit into the avallable space as indicated by the length in the token map.

Xcap (-38) capability error, is returned by Precve when it encounters a capability of protection level higher than that of the bits in the stream.

Xseq $(-101)$ sequence error, is returned when a primitive cannot do its work because another primitive must first be 
invoked. For example, a send may require a preceding obtain, or vice versa; similarly for give and receive. Sequence errors usually arise from programming bugs.

Xref $(-103)$ reject error, is returned when internal waiting by a primitive was terminated by the use of the reject flag, as described above. In general when this error occurs the primitive may have partially but not entirely carried out its purpose, and it may be difficult to continue to use the stream.

Xsid $(-129)$ stream identifier error, is returned by most primitives when an invalid stream identifier is used. However, the getting error, canceling, aborting, and closing primitives are no-ops when supplied an invalid stream identifier, the idea being that such primitives are used for "cleaning up", and an invalid (nonexistent) stream is already "cleaned up"; also the getting parameter primitives have a special meaning for the Null (zero) identifier. Different implementations differ in their ability to recognize an invalid identifier; so a primitive may go ahead in the face of an invalid identifier and do who-knows-what. The only standard is that a Null identifier is always invalid; this gives the user something to store in place of a stream identifier before the stream is opened and after it is closed. An identifier that is valid for some primitives is not valid for all primitives: control transmission should be invalid on a data stream; sending on a receiving stream; etc.

Xerr $(-192)$ transmission error, is returned by any primitive, except the opening and getting-error primitives, when the stream is in a transmission error state, as discussed above.

Xstv (-226) storage overflow error, is returned when a lack of available memory (for internal records or a buffer) prevents a primitive from doing its work.

\section{Interaction with SMILE}

Invisibly to the Application layer, the APST primitives invoke SMILE primitives to obtain memory space for data buffers" and their own internal records. Less invisibly, they rely upon the SMILE tasking and timing primitives. The implementation must provide whatever is needed to communicate transmitted information and task wake-ups across the user-system boundary (if there is one).

The primitives assume that only one task at a time is invoking a primitive for a given stream. Successive primitives on the same stream may be invoked by two or more tasks working together, but if one task invokes a primitive for a stream while another task is executing or waiting within primitive for that same stream, then a sequence error will be returned or perhaps (in less robust implementations) unpredictable behavior will occur. 
Since the APST primitives are defined in terms of the SMILE primitives, the SMILE header file should precede any APST header files in application source files. 


\section{Index}

B bit $3,4,5,13,17,19,21$

big end 4,8

buffer 3

control 4

data 4 21

$E$ bit $3,4,5,10,12,13,16,17,19,20$,

end-code 5

Error Codes

Numeric

$-10121$

$-10321$

$-1221$

$-12921$

$-1421$

$-19222$

$-22622$

$-3421$

$-3521$

$-3621$

$-3721$

$-3821$

$-821$

$-921$

Symbolic

Xbgn 21

Xcap 21

Xend 21

Xerr 22

Xlch 21

XIsn 21

Xrej 21

Xsep 21

Xseq 21

Xsid 21

Xstv 22

Xtyp 21

Xusg 21

Xval 21

Fabs 7.8

Fend 7

Fetc 7

Fimm 6, 8

Find 7

Ftrn 6

go $12,16,21$

LINCS errors 5

little end 4, 8

Mcon 5, 10

mflg 6

mlng 7

Mlob 5,10

Mmul 5, 10

Mrev 5

mtyp 7

musg 7

mval 7

$\mathrm{N}$ bit $5,12,13,17$

Pabort 19

Pcancl 18, 19
Pclose 20

Perror 17, 18

Pgivec 15, 16

Pgived 15, 16

Pinit 20

Pobtnc 13

Pobtnd 13, 14, 16

Popen 9, 10, 20

Pparam 11

Precve 16, 21

Precvd 16, 17, 21

Presentation layer 4

primitives 3

Psendc 12, 13, 15

Psendd 12, 13, 14, 16

rejected 8

Sabort 19

Scancl 19

Sclose 20

Serror 18

Session layer 4

Sgive 15

Sinit 20, 21

Sobtn 14

Sopen 10

Sparam 11

Srecv 17, 21

Ssend 12, 13, 14

stream 3

struct tmap 6

Tabort 19

Tbit 7

Tcancl 19

Tcap 7,8

Tchr 7.8

Tclose 20

Terror 18

Tgive 15

Tinit 20

Tint 7

Tnat 7

Tobtn 14

tokens 4

Topen 10

Tparam 11

transmit now $5,12,13,17$

Transport layer 3

Traw 7,8

Trecv 17

Tsend 13,14

Tvar 7

$W$ bit $3,4,5,12,13,17,19,20,21$ 